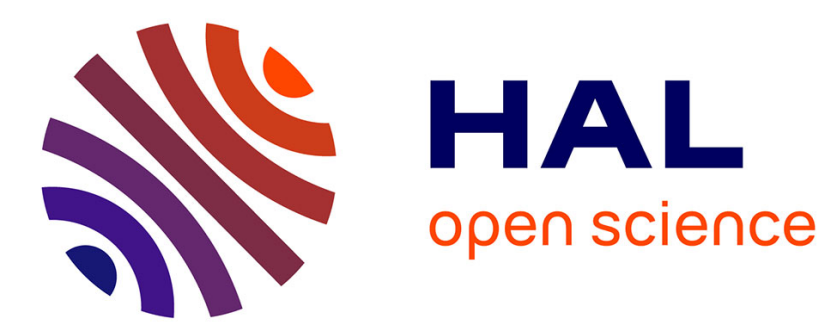

\title{
Dynamical pattern formation in two-dimensional fluids and Landau pole bifurcation
}

Shun Ogawa, Julien Barré, Hidetoshi Morita, Yoshiyuki Y. Yamaguchi

\section{To cite this version:}

Shun Ogawa, Julien Barré, Hidetoshi Morita, Yoshiyuki Y. Yamaguchi. Dynamical pattern formation in two-dimensional fluids and Landau pole bifurcation. Physical Review Online Archive (PROLA), 2014, 89, pp.9. 10.1103/PhysRevE.89.063007 . hal-01086377

\section{HAL Id: hal-01086377 https://hal.science/hal-01086377}

Submitted on 24 Nov 2014

HAL is a multi-disciplinary open access archive for the deposit and dissemination of scientific research documents, whether they are published or not. The documents may come from teaching and research institutions in France or abroad, or from public or private research centers.
L'archive ouverte pluridisciplinaire $\mathbf{H A L}$, est destinée au dépôt et à la diffusion de documents scientifiques de niveau recherche, publiés ou non, émanant des établissements d'enseignement et de recherche français ou étrangers, des laboratoires publics ou privés. 


\title{
Dynamical pattern formations in two dimensional fluid and Landau pole bifurcation
}

\author{
Shun Ogawa,, , * Julien Barré, ${ }^{2}$ Hidetoshi Morita, ${ }^{3,4}$ and Yoshiyuki Y. Yamaguchi ${ }^{1}$ \\ ${ }^{1}$ Department of Applied Mathematics and Physics, Graduate School of Informatics, Kyoto University, 606-8501 Kyoto, Japan \\ ${ }^{2}$ Laboratoire J. A. Dieudonné, Université de Nice Sophia-Antipolis, \\ UMR CNRS 7351, Parc Valrose, F-06108 Nice Cedex 02, France \\ ${ }^{3}$ Department of Mathematics, Kyoto University, 606-8502 Kyoto, Japan \\ ${ }^{4}$ CREST, JST, 606-8502 Kyoto, Japan
}

\begin{abstract}
A phenomenological theory is proposed to analyze the asymptotic dynamics of perturbed inviscid Kolmogorov shear flows in two dimensions. The phase diagram provided by the theory is in qualitative agreement with numerical observations, which include three phases depending on the aspect ratio of the domain and the size of the perturbation: a steady shear flow, a stationary dipole, and four traveling vortices. The theory is based on a precise study of the inviscid damping of the linearized equation and on an analysis of nonlinear effects. In particular, we show that the dominant Landau pole controlling the inviscid damping undergoes a bifurcation, which has important consequences on the asymptotic fate of the perturbation.
\end{abstract}

PACS numbers: 47.54.-r,47.15.ki,05.45.-a

\section{INTRODUCTION}

Patterns in effective two-dimensional (2D) fluids flows are found in nature in various contexts [1, 2]: atmospheric [3, 4] and oceanic flows [5] are examples. To understand such large scale patterns theoretically, the 2D Euler equation describing perfect fluids flows is a simplified starting point. In this context, the study of nonlinear structures, such as Kelvin's cat's eyes, over a shear flow has a long history [6-8], which includes more recent mathematical developments [9, 10].

Statistical physics has often been invoked to explain the formation of large vortices since Onsager [11, 12]. The Miller-Robert-Sommeria (MRS) theory [13-16], which constructs the microcanonical measure for 2D Euler flows by taking all the invariants into account, is a particularly successful achievement. A difficulty in applying this theory in practice is the fact that the 2D Euler equation has infinitely many invariants. More importantly, the theory assumes the vorticity field on the large scales to be stationary, and therefore cannot describe non-stationary asymptotic behaviors. For these cases, the statistical physics approach should be supplemented by a dynamical understanding of pattern formations.

In this paper, we consider a shear base flow to which a perturbation is added, and propose a phenomenological approach to the pattern formations by analyzing the Landau pole of the linearized equation with its bifurcation, and by taking into account nonlinear effects.

Detailed numerical simulations of this situation are presented in Ref. [17]: starting from a Kolmogorov flow on the doubly periodic domain $\mathbb{T}^{2}=[0,2 \pi) \times[0,2 \pi \Gamma)$, a perturbation of size $\epsilon$ is added; depending on the parameters $(\epsilon, \Gamma)$, the perturbation may fully damp, evolve into a stationary dipole, or create four long lived traveling vortices. The regions on the $(\epsilon, \Gamma)$ plane where the dipole or traveling vor-

\footnotetext{
*E-mail: sogawa@amp.i.kyoto-u.ac.jp
}

tices appear are numerically investigated in Ref. [17], but a theoretical understanding is lacking. In this article, we aim at providing such a theoretical explanation, by exploiting the analogy between the 2D Euler equation for the vorticity field and the Vlasov equation for plasmas. Indeed, a similar phenomenon has been investigated in one-dimensional plasmas, described by the Vlasov equation. A naive linear theory predicts that perturbations added to stable stationary states damp exponentially [18]. It is well known, however, that, if the perturbation is large enough, nonlinear effects come into play, prevent complete damping, and may create traveling clusters [19-22]; see also Ref. 23] for the simpler cases of ferromagnetic and anti-ferromagnetic Hamiltonian mean-field model. To be more precise, according to Refs. [19, 23], such a phenomenon occurs when the two following criteria are satisfied:

1. The Landau damping time scale is longer than a nonlinear timescale, called "trapping timescale".

2. If several clusters are formed, they should be so small that they do not overlap and that a nonlinear superposition approximation [24] may work.

Although these criteria are in view of nonlinear dynamics, they can be expressed using the dominant Landau pole computed from the linearized Euler equation. Our goal is to draw the phase diagram in the $(\epsilon, \Gamma)$ plane by using the above two criteria in the context of the Euler equation, that is, by combining the phenomenological nonlinear estimate and the linear Landau damping theory.

This article is organized as follows. In Sec. [I] we qualitatively analyze criteria 1 and 2, and show that they are related to the imaginary and the real parts of the dominant Landau pole of the initial Kolmogorov flow. We therefore briefly review the linear theory for 2D incompressible and inviscid fluids, and derive the dispersion relation in Sec. III the computation of the Landau pole requires an analytic continuation procedure similar to the one used in Ref. [25]. Using these computations, we draw a phase diagram in the $(\epsilon, \Gamma)$ plane in Sec. IV and compare this phenomenological 
estimate with numerical simulations in Sec. $\mathrm{V}$ Section $\mathrm{V}$ is devoted to the summary and discussions.

\section{THE CRITERIA TO OBSERVE A DIPOLE, OR TRAVELING VORTICES}

We start from the 2D Euler equation in the domain $\mathbb{T}^{2}$

$$
\frac{\partial \omega}{\partial t}+\vec{v} \cdot \nabla \omega=0
$$

where the vorticity field $\omega$ and the velocity field $\vec{v}$ are related to a stream function $\psi$ through

$$
\omega=\nabla^{2} \psi, \quad \vec{v}=\left(-\frac{\partial \psi}{\partial y}, \frac{\partial \psi}{\partial x}\right) .
$$

The periods for $x$ and $y$ axes are set as $2 \pi$ and $2 \pi \Gamma$ respectively.

We consider the stationary Kolmogorov flow, called hereafter the "base flow", whose stream function is $\psi_{0}=$ $-\Gamma \sin (y / \Gamma)$ and vorticity and velocity fields are respectively

$$
\omega_{0}(y)=-U^{\prime}(y), \quad \vec{v}_{0}=(U(y), 0),
$$

with $U(y)=\cos (y / \Gamma)$. We add a large scale perturbation to the base flow, and expand the stream function as

$$
\psi(x, y, t)=\psi_{0}(y)+\psi_{1}(x, y, t)
$$

with

$$
\psi_{1}(x, y, t=0)=\epsilon \cos x .
$$

In our analytical computations, $\epsilon$ is assumed to be small; this restriction obviously does not hold for numerical simulations. Nevertheless, the theory will be qualitatively in good agreement with the simulations.

When the base flow is stable (this corresponds to an aspect ratio $\Gamma>1$ ), the linear theory typically predicts that the perturbation damps and possibly oscillates at complex frequency $c=c_{\mathrm{R}}+i c_{\mathrm{I}}$, where $c$ is the root of the dispersion relation yielding the slowest damping, that is, the root closest to the real axis, or, in other words, with the smallest $\left|c_{I}\right|$. The idea is that while it damps, the perturbation will tend to create structures traveling at velocity $c_{R}$; if nonlinear effects are strong enough, these structures may persist for long times. If $c_{\mathrm{R}} \neq 0$, one may expect traveling vortices, while the formation of two stationary vortices (a dipole) should be favored if $c_{\mathrm{R}}=0$.

\section{A. Criterion 1: damping timescale longer than trapping timescale}

The damping time scale $\tau_{\mathrm{L}}$ is easily estimated as the inverse of the Landau damping rate $\tau_{\mathrm{L}} \simeq 1 /\left|c_{\mathrm{I}}\right|$. Actually, in addition to the exponential Landau damping described by $c_{\mathrm{I}}$, there is an algebraic damping coming from the branch points of the dispersion function (see Sec. III); however, the exponential Landau damping is expected to be dominant on the time scale considered in this paper.

Next, we estimate the "trapping time scale", $\tau_{\mathrm{T}}$, which is the characteristic time scale concerning nonlinearities [19], as the period of a test point vortex trapped around the edge of a small vortex. The temporal evolution of the position of a test point vortex is governed by the velocity field

$$
\dot{x}=-\frac{\partial \psi}{\partial y}=U(y)-\frac{\partial \psi_{1}}{\partial y}, \quad \dot{y}=\frac{\partial \psi}{\partial x}=\frac{\partial \psi_{1}}{\partial x},
$$

where the dot denotes $\mathrm{d} / \mathrm{d} t$. We approximate the perturbation $\psi_{1}(x, y, t)$ phenomenologically. We are interested in the macroscopic behavior corresponding to the $k= \pm 1$ mode, where $k$ is the wave number with respect to $x$, and we assume that the damping perturbation has created small vortices whose velocity is $c_{\mathrm{R}}$ in the $x$ direction; note that $c_{\mathrm{R}}$ can be 0 . The first order $\psi_{1}(x, y, t)$ can be, therefore, approximated by

$$
\psi_{1}(x, y, t) \simeq \epsilon \hat{\psi}_{1}(y) \cos \left(x-c_{\mathrm{R}} t\right), \quad U(y) \sim c_{\mathrm{R}},
$$

where $\hat{\psi}_{1}(y)$ is of $O\left(\epsilon^{0}\right)$. Substituting Eq. (7) into Eq. [6], we have the approximate equations of motion

$$
\begin{aligned}
& \dot{x}=U(y)-\epsilon \hat{\psi}_{1}^{\prime}(y) \cos \left(x-c_{\mathrm{R}} t\right), \\
& \dot{y}=-\epsilon \hat{\psi}_{1}(y) \sin \left(x-c_{\mathrm{R}} t\right),
\end{aligned}
$$

where the prime represents $\mathrm{d} / \mathrm{d} y$. Thanks to the phenomenological approximation of $\psi_{1}$ (7), we can compute the motion of the test point vortex by perturbation techniques. Expanding $x$ and $y$ into series of $\epsilon$ as

$$
\begin{aligned}
& x(t)=x_{0}(t)+\epsilon x_{1}(t)+O\left(\epsilon^{2}\right), \\
& y(t)=y_{0}(t)+\epsilon y_{1}(t)+O\left(\epsilon^{2}\right),
\end{aligned}
$$

we obtain the solutions $x_{0}(t)$ and $y_{0}(t)$ with initial conditions $x_{0}(0)=X$ and $y_{0}(0)=Y$ :

$$
x_{0}(t)=X+U(Y) t, \quad y_{0}(t)=Y
$$

Equations for the first order in $\epsilon$ are:

$$
\begin{aligned}
& \dot{x}_{1}(t)=U^{\prime}(Y) y_{1}(t)-\hat{\psi}_{1}^{\prime}(Y) \cos \left(X+\left(U(Y)-c_{\mathrm{R}}\right) t\right), \\
& \dot{y}_{1}(t)=-\hat{\psi}_{1}(Y) \sin \left(X+\left(U(Y)-c_{\mathrm{R}}\right) t\right) .
\end{aligned}
$$

From the frequency of $y_{1}$ in Eq. [11, we estimate the trapping time scale $\tau_{\mathrm{T}}$ as

$$
\tau_{\mathrm{T}} \simeq \frac{1}{\left|U\left(Y_{*}+g(\epsilon, \Gamma)\right)-c_{\mathrm{R}}\right|} \simeq \frac{1}{g(\epsilon, \Gamma)\left|U^{\prime}\left(Y_{*}\right)\right|}
$$

where $Y_{*}$ satisfies $U\left(Y_{*}\right)=c_{\mathrm{R}}$, and $g(\epsilon, \Gamma)>0$ represents the width of a vortex.

The unknown quantity, the width of a vortex $g(\epsilon, \Gamma)$, is determined self-consistently. The solution $y_{1}(t)$ is, from Eq. 
[11,

$$
y_{1}(t)=\frac{\hat{\psi}_{1}(Y)}{U(Y)-c_{\mathrm{R}}}\left[\cos \left(X+\left(U(Y)-c_{\mathrm{R}}\right) t\right)-\cos X\right],
$$

where the initial condition is $y_{1}(0)=0$. The amplitude in the $y$ direction, $\epsilon \hat{\psi}_{1}(Y) /\left(U(Y)-c_{\mathrm{R}}\right)$, must be the same as the width $g(\epsilon, \Gamma)$ at the edge of the vortex, $Y=Y_{*}+g$. Thus, the self-consistent equation for $g$ is

$$
g=\frac{\epsilon}{U\left(Y_{*}+g\right)-c_{\mathrm{R}}},
$$

where we have introduced another phenomenological approximation by replacing $\hat{\psi}_{1}(Y)$ with 1 , since it is of $O\left(\epsilon^{0}\right)$ and we are looking for an order-of-magnitude estimate. The width $g$ is, therefore, estimated as

$$
g(\epsilon, \Gamma) \approx \sqrt{\frac{\epsilon}{\left|U^{\prime}\left(Y_{*}\right)\right|}}
$$

For the Kolmogorov base flow, $U(y)=\cos (y / \Gamma)$, we have

$$
\left|U^{\prime}\left(Y_{*}\right)\right|=\frac{1}{\Gamma} \sqrt{1-c_{\mathrm{R}}^{2}},
$$

and the trapping time scale is

$$
\tau_{\mathrm{T}}=\sqrt{\frac{\Gamma}{\epsilon}}\left(1-c_{\mathrm{R}}^{2}\right)^{-1 / 4} .
$$

Criterion 1 reads $\tau_{\mathrm{T}}<\tau_{\mathrm{L}}$, that is:

$$
\frac{\Gamma c_{\mathrm{I}}^{2}}{\sqrt{1-c_{\mathrm{R}}^{2}}}<\epsilon .
$$

If $c_{\mathrm{R}}=0$, this condition simplifies into $\Gamma c_{\mathrm{I}}^{2}<\epsilon$.

\section{B. Criterion 2: non overlapping vortices}

According to Eq. (5), we consider the modes $k= \pm 1$. We assume that the frequency $c_{\mathrm{R}}$ of the perturbation reflects the existence of periodically moving vortices, thanks to the periodic boundary condition for the $x$ axis. The period $2 \pi$ permits to identify the frequency with the velocity of the moving vortices, and therefore the $y$ positions of the vortices are estimated as the solutions of the equation $U(y)= \pm c_{\mathrm{R}}$. We recall the base flow is $U(y)=\cos (y / \Gamma)$.

If $c_{\mathrm{R}}=0$, one expects that vortices are formed at $y=\pi \Gamma / 2$ and $y=3 \pi \Gamma / 2$. The estimate of the vortices width (15) shows that the vortices will never overlap for any reasonably small $\epsilon$ (say for instance $\epsilon<0.5$ ). Hence criterion 2 brings no restriction in this case.

If $c_{\mathrm{R}} \neq 0$, in contrast, the vortices can be close one to another, and criterion 2 leads to a restriction. We name the four vortices A, B, C, and D, whose $y$ positions are, respectively, $\Gamma \arccos \left(\left|c_{R}\right|\right), \pi \Gamma-\Gamma \arccos \left(\left|c_{R}\right|\right), \pi \Gamma+\Gamma \arccos \left(\left|c_{R}\right|\right)$ and $2 \pi \Gamma-\Gamma \arccos \left(\left|c_{R}\right|\right)$ in the interval $[0,2 \pi \Gamma)$, where we

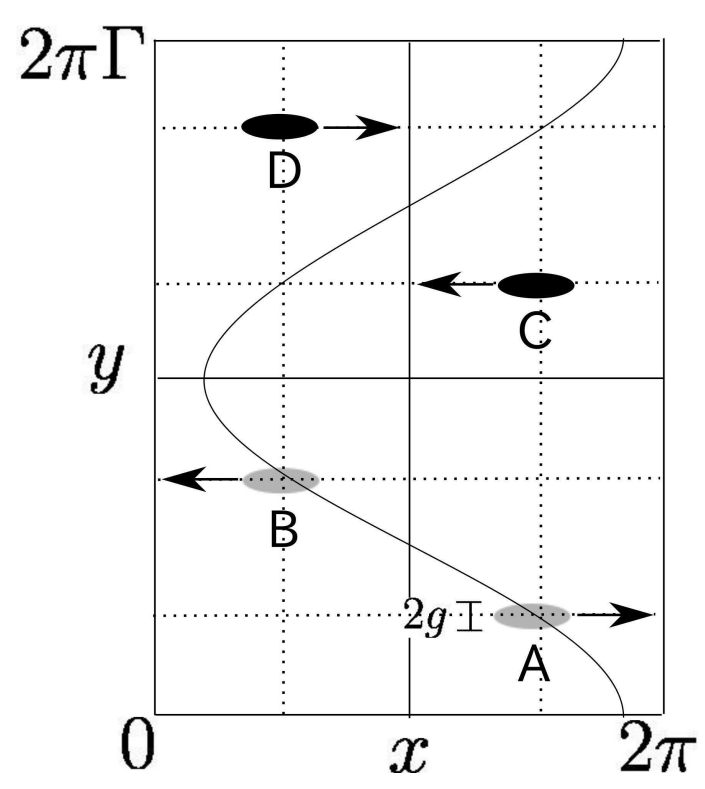

FIG. 1. Schematic picture of four traveling vortices, A, B, C, and D. The arrows indicate traveling directions. Vortices A and B have positive vorticity, while $\mathrm{C}$ and $\mathrm{D}$ negative. The solid curve represents the velocity of the base flow $U(y)$, suitably rescaled. The two vertical dotted lines correspond to $\pm c_{\mathrm{R}}$; their intersections with the velocity curve, $U(y)= \pm c_{\mathrm{R}}$, yield the estimated $y$ positions of the four vortices, which travel along the horizontal dotted lines.

take the branch of solutions $0 \leq \arccos \left(\left|c_{\mathrm{R}}\right|\right) \leq \pi / 2$; see Fig. 1]

We assume that each vortex has the same width $g$. Then the distance between two nearby vortices must be larger than $2 g$ in order to avoid an overlap. Using $\pi / 2-$ $\arccos \left(c_{\mathrm{R}}\right)=\arcsin \left(c_{\mathrm{R}}\right)$, this condition is expressed as

$$
\begin{cases}\Gamma \arcsin \left(c_{R}\right)>g, & \left(\left|c_{R}\right|<1 / \sqrt{2}\right), \\ \Gamma \arccos \left(c_{R}\right)>g, & \left(\left|c_{R}\right|>1 / \sqrt{2}\right) .\end{cases}
$$

The first inequality comes from the distance in $y$ between vortices A and B (identically $\mathrm{C}$ and $\mathrm{D}$ ), and the second from the distance between $B$ and $C$ (identically $A$ and $D$ ). The pair $(\mathrm{B}, \mathrm{C})$ moves toward the left while $(\mathrm{A}, \mathrm{D})$ toward the right according to the base flow $U(y)$. Moreover, the numerical observations [17] say that the difference in $x$ among each pair stays around $\pi$. Thus the distance between B and C (A and D) is large enough to neglect the effect of overlapping; see Fig. 1. Hence we can omit the second inequality of Eq. (19), and criterion 2 is finally expressed as:

$$
\epsilon<\Gamma \sqrt{1-c_{\mathrm{R}}^{2}}\left(\arcsin \left(c_{\mathrm{R}}\right)\right)^{2} .
$$

The two conditions (18) and (20) involve the parameters $\Gamma$ and $\epsilon$, as well as the dominant Landau pole $c=c_{\mathrm{R}}+i c_{\mathrm{I}}$. In the following section, we turn to the computation of this Landau pole. 


\section{THE LINEAR THEORY AND THE DISPERSION RELATION}

This section contains a classical computation for the linearized 2D Euler equation, as well as an analytic continuation in the spirit of Ref. [25], for the self-consistency of the paper. We perform it for a general base flow and perturbation, and then specialize it to Eqs. (3) and (5).

\section{A. The dispersion function $D(c)$}

We linearize Eqs. (1) and (2) around the base flow (3). We add a small perturbation $\psi_{1}$ to $\psi_{0}(y)$, whose associated vorticity and velocity fields are denoted respectively as $\omega_{1}$ and $\vec{v}_{1}$. Substituting $\omega=\omega_{0}+\omega_{1}$ and $\vec{v}=\vec{v}_{0}+\vec{v}_{1}$ into the Euler equation (1) gives the linearized 2D Euler equation

$$
\frac{\partial \omega_{1}}{\partial t}+\vec{v}_{0} \cdot \nabla \omega_{1}+\vec{v}_{1} \cdot \nabla \omega_{0}=0
$$

Using the perturbation $\psi_{1}$ and Eq. 3, we rewrite the linearized Euler equation 21] as

$$
\frac{\partial \omega_{1}}{\partial t}+U(y) \frac{\partial \omega_{1}}{\partial x}-U^{\prime \prime}(y) \frac{\partial \psi_{1}}{\partial x}=0 .
$$

Our goal is now to derive the dispersion relation for the linearized Euler equation; we use a Fourier-Laplace transformation, and follow the route of Ref. [26]. Thanks to the periodicity with respect to $x$, we expand $\omega_{1}$ and $\psi_{1}$ into Fourier series as

$$
\begin{aligned}
& \omega_{1}(x, y, t)=\sum_{k \in \mathbb{Z}} \hat{\omega}_{k}(y, t) e^{i k x}, \\
& \psi_{1}(x, y, t)=\sum_{k \in \mathbb{Z}} \hat{\psi}_{k}(y, t) e^{i k x} .
\end{aligned}
$$

Substituting the Fourier expansions 23 into the linearized Euler equation (22), we obtain the equation for $k$-th Fourier mode as

$$
\frac{\partial \hat{\omega}_{k}}{\partial t}+i k U(y) \hat{\omega}_{k}-i k \hat{\psi}_{k} U^{\prime \prime}(y)=0, \quad \forall k \in \mathbb{Z} .
$$

The Laplace transform of a function $\hat{g}(t)$ with respect to $t$ is defined as

$$
\tilde{g}(z)=\int_{0}^{\infty} \hat{g}(t) e^{-z t} d t, \quad \operatorname{Re} z>0,
$$

where the condition $\operatorname{Re} z>0$ is introduced to ensure the convergence of the integral. Performing the Laplace transform of the linearized Euler equation (24), we obtain an algebraic equation for $\tilde{\omega}_{k}$ and $\tilde{\psi}_{k}$ :

$$
(U(y)-c) \tilde{\omega}_{k}-U^{\prime \prime}(y) \tilde{\psi}_{k}=\frac{\hat{\omega}_{k}(y, 0)}{i k},
$$

where $z=-i k c$; note that $\operatorname{Im}(k c)>0$.
The Fourier transform of Eq. 22 with respect to $x$

$$
\tilde{\omega}_{k}(y, c)=\left(\frac{\partial^{2}}{\partial y^{2}}-k^{2}\right) \tilde{\psi}_{k}(y, c)
$$

gives a closed equation for $\tilde{\psi}_{k}(y, c)$, which is the Rayleigh equation

$$
\frac{\partial^{2} \tilde{\psi}_{k}}{\partial y^{2}}-k^{2} \tilde{\psi}_{k}-\frac{U^{\prime \prime}(y)}{U(y)-c} \tilde{\psi}_{k}=\frac{\hat{\omega}_{k}(y, 0)}{i k(U(y)-c)} .
$$

The stream function $\hat{\psi}_{k}(y, t)$ is computed through an inverse Laplace transform. Its asymptotic behavior is determined by the singularities of $\tilde{\psi}_{k}(y, c)$ with respect to the complex variable $c$. For simplicity, we introduce the functions $q$ and $f$ as

$$
q(y) \equiv k^{2}+\frac{U^{\prime \prime}(y)}{U(y)-c}, \quad f(y) \equiv \frac{\hat{\omega}_{k}(y, 0)}{i k(U(y)-c)} .
$$

These functions have no singularity for real $y$, since $c$ is defined in the region $\operatorname{Im}(k c)>0$.

Fixing the complex variable $c$, we analyze the Rayleigh equation of the form

$$
\frac{\mathrm{d}^{2} \phi}{\mathrm{d} y^{2}}-q(y) \phi=f(y),
$$

and the corresponding homogeneous Rayleigh equation

$$
\frac{\mathrm{d}^{2} \phi}{\mathrm{d} y^{2}}-q(y) \phi=0 .
$$

Let $\phi_{1}$ and $\phi_{2}$ be independent solutions to the homogeneous equation 31 with boundary conditions

$$
\left\{\begin{array} { l } 
{ \phi _ { 1 } ( 0 ) = 1 , } \\
{ \phi _ { 1 } ^ { \prime } ( 0 ) = 0 , }
\end{array} \text { and } \left\{\begin{array}{l}
\phi_{2}(0)=0, \\
\phi_{2}^{\prime}(0)=1 .
\end{array}\right.\right.
$$

The particular solution $\phi_{\mathrm{p}}$ to the inhomogeneous equation (30) is then given by

$$
\begin{aligned}
\phi_{\mathrm{p}}(y)=-\phi_{1}(y) & \int_{0}^{y} \phi_{2}\left(y^{\prime}\right) f\left(y^{\prime}\right) d y^{\prime} \\
& +\phi_{2}(y) \int_{0}^{y} \phi_{1}\left(y^{\prime}\right) f\left(y^{\prime}\right) d y^{\prime}
\end{aligned}
$$

Indeed, the double derivative of $\phi_{\mathrm{p}}(y)$ is

$$
\phi_{\mathrm{p}}^{\prime \prime}(y)=q(y) \phi_{\mathrm{p}}(y)+W(y) f(y)
$$

where $W(y)$ is the Wronskian

$$
W(y)=\phi_{1}(y) \phi_{2}^{\prime}(y)-\phi_{1}^{\prime}(y) \phi_{2}(y)
$$

and is constant

$$
W(y)=W(0)=1 \text { for all } y
$$


Hence, the general solution $\phi_{\mathrm{g}}$ to Eq. 28] is:

$$
\phi_{\mathrm{g}}=\phi_{\mathrm{p}}+a_{1} \phi_{1}+a_{2} \phi_{2},
$$

where $a_{1}$ and $a_{2}$ are constants determined from the periodic boundary condition

$$
\phi_{\mathrm{g}}(2 \pi \Gamma)=\phi_{\mathrm{g}}(0), \quad \phi_{\mathrm{g}}^{\prime}(2 \pi \Gamma)=\phi_{\mathrm{g}}^{\prime}(0) .
$$

From the boundary conditions 32 and $\phi_{\mathrm{p}}(0)=\phi_{\mathrm{p}}^{\prime}(0)=0$, Eqs. (37) and (38) lead to:

$$
\left(\begin{array}{cc}
\phi_{1}(2 \pi \Gamma)-1 & \phi_{2}(2 \pi \Gamma) \\
\phi_{1}^{\prime}(2 \pi \Gamma) & \phi_{2}^{\prime}(2 \pi \Gamma)-1
\end{array}\right)\left(\begin{array}{l}
a_{1} \\
a_{2}
\end{array}\right)=-\left(\begin{array}{l}
\phi_{\mathrm{p}}(2 \pi \Gamma) \\
\phi_{\mathrm{p}}^{\prime}(2 \pi \Gamma)
\end{array}\right) .
$$

Remembering that $q(y)$ and hence $\phi_{1}$ and $\phi_{2}$ depend on $c$, we define the function $D(c)$ as

$$
\begin{aligned}
D(c) & =\operatorname{det}\left(\begin{array}{cc}
\phi_{1}(2 \pi \Gamma)-1 & \phi_{2}(2 \pi \Gamma) \\
\phi_{1}^{\prime}(2 \pi \Gamma) & \phi_{2}^{\prime}(2 \pi \Gamma)-1
\end{array}\right) \\
& =2-\phi_{1}(2 \pi \Gamma)-\phi_{2}^{\prime}(2 \pi \Gamma) .
\end{aligned}
$$

To show the last equality in Eq. 40, we have used the fact that the Wronskian $W(y)$ is unity. The values of $\phi_{1}(2 \pi \Gamma)$ and $\phi_{2}^{\prime}(2 \pi \Gamma)$ are computed by integrating the homogeneous Rayleigh equation (31) from 0 to $2 \pi \Gamma$.

\section{B. Analytic continuation of $D(c)$}

The general solution (37) has singularities for $c$ satisfying $D(c)=0$, and these singularities yield non-trivial modes proportional to $e^{-i k c t}$ by the inverse Laplace transform. This justifies our terminology "dispersion relation" for $D(c)=0$, and "dispersion function" for the function $D(c)$. Recall that the dispersion function $D(c)$ is a priori defined only in the region $\operatorname{Im}(k c)>0$ to ensure the convergence of the Laplace transform. Now, to find roots giving stable modes, we analytically continue $D(c)$ to the whole complex $c$ plane, in a similar manner to what has been performed for the Euler equation on the 2D disc [25, 27, 28].

Hereafter we set $k=1$ without loss of generality, and take the particular form of the base flow: $U(y)=\cos (y / \Gamma)$. Corresponding to a given $c$, the functions $q(y)$ and $f(y)$ have singularities at which the equation $U(y)-c=0$ is satisfied. Such singular points are, together with the integration paths of the homogeneous Rayleigh equation [31, schematically illustrated in Fig. 22 for both the cases $\operatorname{Im} c>0$ and $\operatorname{Im} c<0$. The integration paths are determined as follows.

For $c$ on the upper half plane, $D(c)$ is simply defined by integrating Eq. (31) along the real $y$ axis, namely along the integration path:

$$
L=\{y \in \mathbb{R} \mid y=y(s)=2 \pi \Gamma s, s \in[0,1)\} .
$$

Continuously moving $c$ to the lower half plane allows to define $D(c)$ for $\operatorname{Im} c<0$. If the path taking $c$ from the upper to the lower half plane crosses the singular interval $c \in[-1,1]$, we have to avoid the singularity that would be created. For this purpose, we deform the integration path $L$ to

$$
L_{h}=\left\{y \in \mathbb{C} \mid y=y_{h}(s)=2 \pi \Gamma(s+i h(s)), s \in[0,1)\right\},
$$

using a $C^{2}$-class, real-valued, and 1-periodic function $h(s)$ satisfying $h(0)=h(1)=0$; correspondingly, this amounts to deform the singular line

$$
\sigma=\{c \in \mathbb{R} \mid c=U(y(s)), s \in[0,1)\},
$$

which doubly covers the interval $[-1,1]$, to the curve

$$
\sigma_{h}=\left\{c \in \mathbb{C} \mid c=U\left(y_{h}(s)\right), s \in[0,1)\right\} .
$$

We choose the function $h(s)$ so that the deformed curve $\sigma_{h}$ is below the $c$ for which we want to define $D(c)$. This procedure is similar to the spectral deformation for the VlasovPoisson equation in Refs. [29, 30].

If the path taking $c$ from the upper to the lower plane crosses the real axis through either one of the half lines $(-\infty,-1)$ or $(1, \infty)$, no singularity is crossed, and the analytic continuation does not require any deformation of the integral path; in the lower half plane, $D(c)$ is multi valued unless a Riemann surface associated with the branch points $c= \pm 1$ is introduced.

We choose the particular deformation function $h(s)$ in Eq. (42) as

$$
h(s)=a \sin 2 \pi s, \quad a \geq 0 .
$$

The extended domains of $D(c)$ are shown in Fig. 3 for two different values of $a$. We use $a$ large enough so that $\sigma_{h}$ is below $c$; the computational cost increases exponentially with increasing $a$.

By introducing the following functions of $s$ :

$$
\varphi(s)=\phi\left(y_{h}(s)\right), \quad p(s)=q\left(y_{h}(s)\right),
$$

the homogeneous Rayleigh equation (31) is rewritten as

$$
\begin{aligned}
\varphi_{\mathrm{R}}^{\prime \prime} & -\frac{h^{\prime} h^{\prime \prime}}{1+h^{\prime 2}} \varphi_{\mathrm{R}}^{\prime}+\frac{h^{\prime \prime}}{1+h^{2}} \varphi_{\mathrm{I}}^{\prime} \\
& -(2 \pi \Gamma)^{2}\left(\left(1-h^{\prime 2}\right) p_{\mathrm{R}}-2 h^{\prime} p_{\mathrm{I}}\right) \varphi_{\mathrm{R}} \\
& +(2 \pi \Gamma)^{2}\left(2 h^{\prime} p_{\mathrm{R}}+\left(1-h^{\prime 2}\right) p_{\mathrm{I}}\right) \varphi_{\mathrm{I}}=0, \\
\varphi_{\mathrm{I}}^{\prime \prime} & -\frac{h^{\prime \prime}}{1+h^{\prime 2}} \varphi_{\mathrm{R}}^{\prime}-\frac{h^{\prime} h^{\prime \prime}}{1+h^{\prime 2}} \varphi_{\mathrm{I}}^{\prime} \\
& -(2 \pi \Gamma)^{2}\left(2 h^{\prime} p_{\mathrm{R}}+\left(1-h^{\prime 2}\right) p_{\mathrm{I}}\right) \varphi_{\mathrm{R}} \\
& -(2 \pi \Gamma)^{2}\left(\left(1-h^{\prime 2}\right) p_{\mathrm{R}}-2 h^{\prime} p_{\mathrm{I}}\right) \varphi_{\mathrm{I}}=0,
\end{aligned}
$$

where the subscripts "R" and "I" represent the real and imaginary parts respectively, and the prime denotes the derivative with respect to $s$. The continued solutions $\varphi_{1}$ and $\varphi_{2}$ are computed by solving Eq. 477 with the boundary con- 


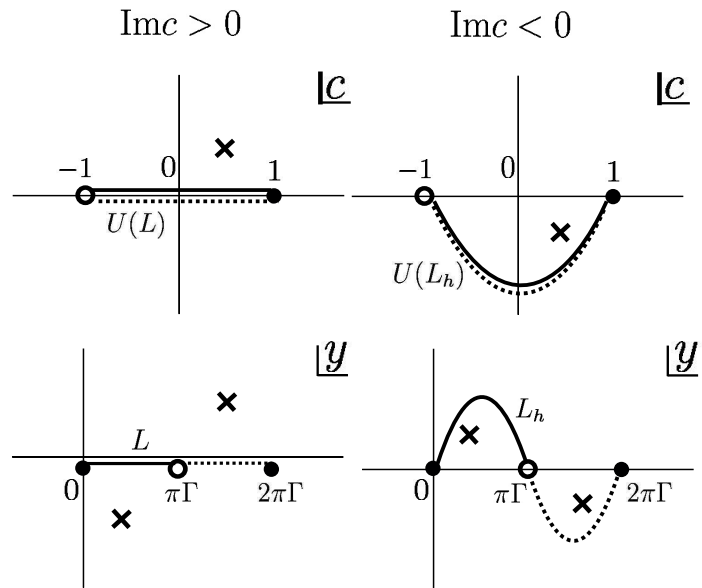

FIG. 2. Schematic picture of complex $c$ and $y$ planes for $k=1$. The left two panels are for $\operatorname{Im} c>0$, and the right two panels for $\operatorname{Im} c<0$. The integration path $L$ on the $y$ plane, consisting of the solid and the dotted lines, is mapped to the double covering of the interval $[-1,1]$ on the $c$ plane by the mapping $U: y \mapsto U(y)$. Corresponding to a given cross point on the $c$ plane, there are two singular points (crosses) of $q(y)$ and $f(y)$ on the $y$ plane in general. As the point on the $c$ plane goes down, the singular points on the $y$ plane pass the real axis and the integration path $L$ must be deformed to $L_{h}$ to avoid the singularities. The mapping of $L_{h}$, denoted by $U\left(L_{h}\right)$, determines the boundary of the continued domain of the dispersion function $D(c)$. See Fig. 3 A branch cut on the $c$ plane is set as the boundary.

ditions

$$
\left\{\begin{array} { l } 
{ \varphi _ { 1 , \mathrm { R } } ( 0 ) = 1 , } \\
{ \varphi _ { 1 , \mathrm { I } } ( 0 ) = 0 , } \\
{ \varphi _ { 1 , \mathrm { R } } ^ { \prime } ( 0 ) = 0 , } \\
{ \varphi _ { 1 , \mathrm { I } } ^ { \prime } ( 0 ) = 0 , }
\end{array} \text { and } \quad \left\{\begin{array}{l}
\varphi_{2, \mathrm{R}}(0)=0, \\
\varphi_{2, \mathrm{I}}(0)=0, \\
\varphi_{2, \mathrm{R}}^{\prime}(0)=2 \pi \Gamma \\
\varphi_{2, \mathrm{I}}^{\prime}(0)=2 \pi \Gamma h^{\prime}(0)
\end{array}\right.\right.
$$

respectively. Solving Eq. 477, we obtain $\varphi_{1}(1)$ and $\varphi_{2}^{\prime}(1)$, and the dispersion function is expressed as

$$
D(c)=2-\varphi_{1}(1)-\frac{\varphi_{2}^{\prime}(1)}{2 \pi \Gamma\left(1+i h^{\prime}(1)\right)},
$$

whose real and imaginary parts are respectively

$$
\begin{aligned}
& D_{\mathrm{R}}(c)=2-\varphi_{1, \mathrm{R}}(1)-\frac{\varphi_{2, \mathrm{R}}(1)+h^{\prime}(1) \varphi_{2, \mathrm{I}}(1)}{2 \pi \Gamma\left(1+h^{\prime}(1)^{2}\right)}, \\
& D_{\mathrm{I}}(c)=-\varphi_{1, \mathrm{I}}(1)+\frac{h^{\prime}(1) \varphi_{2, \mathrm{R}}(1)-\varphi_{2, \mathrm{I}}(1)}{2 \pi \Gamma\left(1+h^{\prime}(1)^{2}\right)} .
\end{aligned}
$$

\section{The main Landau pole and its bifurcation}

We now specialize the above computations to the initial perturbation

$$
\omega_{1}(x, y, 0)=-\epsilon \cos x,
$$

(a) $a=0.1$

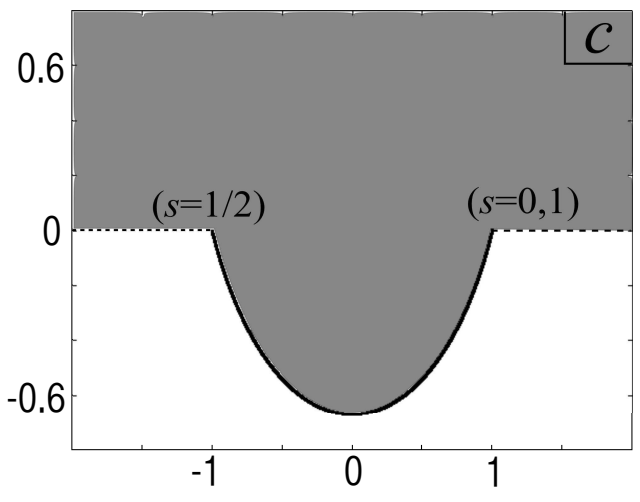

(b) $a=0.3$

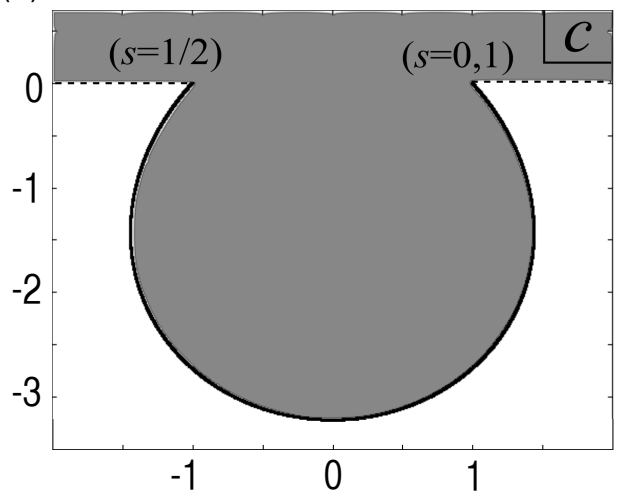

FIG. 3. Extension of the domain of $D(c)$. The gray regions represent the domains of $D(c)$ for (a) $a=0.1$ and (b) $a=0.3$. The curve in the $c$ plane represents $U\left(y_{h}(s)\right)$, where $y_{h}(s)$ is the deformed integration path for the homogeneous Rayleigh equation 31. With increasing $a$, the domain of $D(c)$ is extended to a larger area in the lower half $c$ plane, but the computational cost also increases.

as in the numerics. Using the analytical continuation of $D(c)$ into the lower half plane, we numerically compute Landau poles, i.e. the roots of the equation $D(c)=0$ with $\operatorname{Im} c<0$. We are interested in the traveling vortices, whose velocity is $c_{\mathrm{R}}$, in resonance to the base flow, whose velocity $U(y)$ is in the range $[-1,1]$. It is therefore natural to choose the analytical continuation of $D(c)$ defined by deforming the integral path as in Eq. 45].

We look for the dominant Landau pole, i.e., the root of $D(c)$ with the largest imaginary part; in a stable situation, it corresponds to the slowest damping. The variation of this pole as a function of the aspect ratio $\Gamma$ is shown on Fig. 4 For $\Gamma<1$, the flow is unstable. With increasing $\Gamma$ above 1 , the flow becomes stable, with the main Landau pole on the imaginary axis. With further increasing $\Gamma$, the dominant Landau pole undergoes a bifurcation at an aspect ratio $\Gamma_{\mathrm{c}} \simeq 1.06$ and acquires a non-zero real part. We will see that this bifurcation is crucial to understand the appearance of a stationary dipole or of the four traveling vortices. 


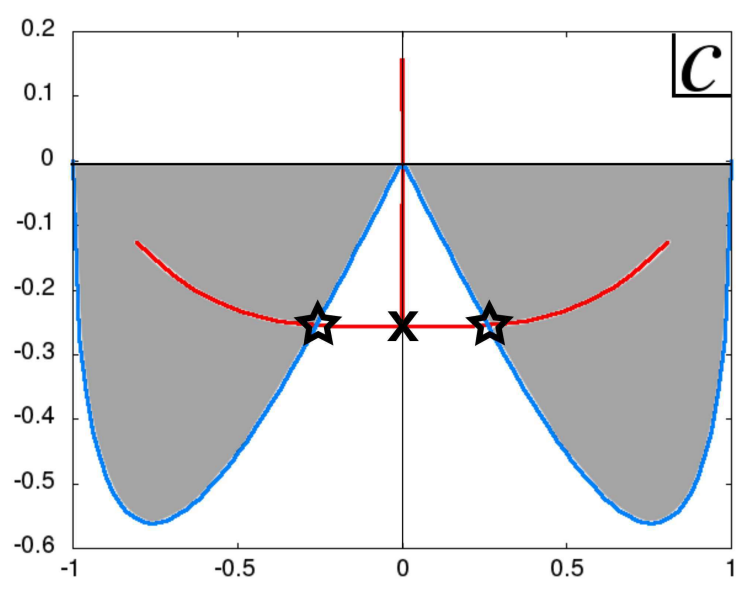

FIG. 4. (color online) The dominant root of the dispersion function (red line) and a favorable region for the existence of traveling vortices (gray region) on the complex $c$ plane. When $\Gamma<1$, one dominant root is obtained on the upper half plane, where the base flow is unstable, and the root is at the origin for $\Gamma=1$. With increasing $\Gamma$, the main root goes down to the lower half plane along the imaginary $c$ axis, accompanied by another root going up the axis. When $\Gamma=\Gamma_{\mathrm{c}} \simeq 1.06$, these two roots collide at $c=-0.28 i$ and they bifurcate to the right and left directions. The gray region is given by the inequality, $c_{\mathrm{I}}^{2}<\left(1-c_{\mathrm{R}}^{2}\right)\left(\arcsin \left(c_{\mathrm{R}}\right)\right)^{2}$ coming from Eq. [52, and $c_{\mathrm{I}}<0$ required by the stability of the flow. For later convenience, we marked the bifurcation point corresponding $\Gamma=\Gamma_{\mathrm{c}}$ by the cross, and the point at which the main pole enters the gray region by thestars.

\section{THE PHASE DIAGRAM}

Using the theoretical analyses above, we construct the phase diagram on the $(\epsilon, \Gamma)$ plane, which consists of zonal, dipole and oscillation phases [17]. The last oscillation phase corresponds to the four traveling vortices.

To obtain the region on the $(\epsilon, \Gamma)$ plane where the appearance of four traveling vortices is expected, we combine criteria 1 and 2, that is Eqs. (18) and 20 respectively, with the result on the dominant Landau pole. Satisfying both criteria then reads:

$$
\frac{\Gamma c_{\mathrm{I}}^{2}}{\sqrt{1-c_{\mathrm{R}}^{2}}}<\epsilon<\Gamma \sqrt{1-c_{\mathrm{R}}^{2}}\left(\arcsin \left(c_{\mathrm{R}}\right)\right)^{2} .
$$

The right inequality requires a Landau pole with non-zero $c_{\mathrm{R}}$, which implies $\Gamma>\Gamma_{\mathrm{c}}$. The region which satisfies Eq. [52] on the $(\epsilon, \Gamma)$ plane is reported in Fig. 5. which is qualitatively in good agreement with the numerically obtained oscillatory region [17]. We remark that $\left|c_{R}\right|$ increases when $\Gamma$ is increased above $\Gamma_{\mathrm{c}}$, staying smaller than $1 / \sqrt{2}$ in the reported parameter region. This fact validates that we have omitted the lower condition in Eq. (19) to derive the second necessary condition.

We expect to find a stationary dipole when criterion 1 is satisfied and $c_{\mathrm{R}}=0$ :

$$
\Gamma c_{\mathrm{I}}^{2}<\epsilon \text { and } \Gamma<\Gamma_{\mathrm{c}} .
$$

The region satisfying these requirements is highlighted in Fig. 5. This theoretically estimated region is again in qualitative agreement with the region where a stationary dipole emerges in numerical simulations [17.

We expect a zonal flow when criterion 1 is not satisfied, that is

$$
\begin{array}{r}
\Gamma c_{\mathrm{I}}^{2}>\epsilon, \text { for } \Gamma<\Gamma_{\mathrm{c}}, \\
\frac{\Gamma c_{\mathrm{I}}^{2}}{\sqrt{1-c_{\mathrm{R}}^{2}}}>\epsilon, \text { for } \Gamma>\Gamma_{\mathrm{c}} .
\end{array}
$$

Physically, it means that nonlinearity is not dominant and hence simple damping of the perturbation is expected. In Fig. 5the zonal region appears for small $\epsilon$.

On Fig. 5 there is a region where the theory makes no prediction; indeed, the vortices that would be created by the phenomenological mechanism considered above would be so close one to another that they would strongly interact; the final fate of the system is then out of scope of the present theory. We note that in this region, zonal flows, dipoles and traveling vortices are observed numerically [17].

\section{NUMERICAL TESTS}

According to the phenomenological prediction of the preceding sections, the four traveling vortices run in the $x$ direction along the four lines $y=y_{*}$ in $\mathbb{T}^{2}=[0,2 \pi) \times[0,2 \pi \Gamma)$, where $y_{*}=\Gamma \arccos \left( \pm c_{\mathrm{R}}\right)$, and their velocities are $U\left(y_{*}\right)=$ $\pm c_{\mathrm{R}}$. The period in the $x$ direction is $2 \pi$; hence the phenomenological theory predicts the frequency $f_{\mathrm{pt}}=\left|c_{\mathrm{R}}\right| / 2 \pi$ for the vorticity field $\omega$.

We examine our phenomenological prediction by numerically observing the $y$-positions of the four vortices and the oscillation frequency. The initial condition of the vorticity field is:

$$
\omega(x, y, 0)=\omega_{0}(x, y)-\epsilon \cos x
$$

fixing the small parameter $\epsilon=0.3$. In numerical simulations, we add a hyper-viscous term $(-1)^{h+1} v \nabla^{2 h} \omega$ to the right hand side of the $2 \mathrm{D}$ euler equation, with $h=4$ and $v=2 \cdot 10^{-18}$, for stabilizing the numerical scheme, and use the pseudo-spectral method with the resolution $256 \times 256$.

First, we observe the $y$-positions by computing the averaged vorticity field $\bar{\omega}(y)$ defined by

$$
\bar{\omega}(y, t)=\frac{1}{2 \pi} \int_{0}^{2 \pi} \omega(x, y, t) d x .
$$

At the initial time $t=0$, the averaged vorticity field is $\omega_{0}(y)=$ $\sin (y / \Gamma) / \Gamma$, and it evolves in time. Asymptotically, we observe four bumps in $\bar{\omega}(y)$ as shown in Fig. 6 for $\Gamma=1.4$ and $\epsilon=0.3$. The theoretical prediction is in good agreement with 

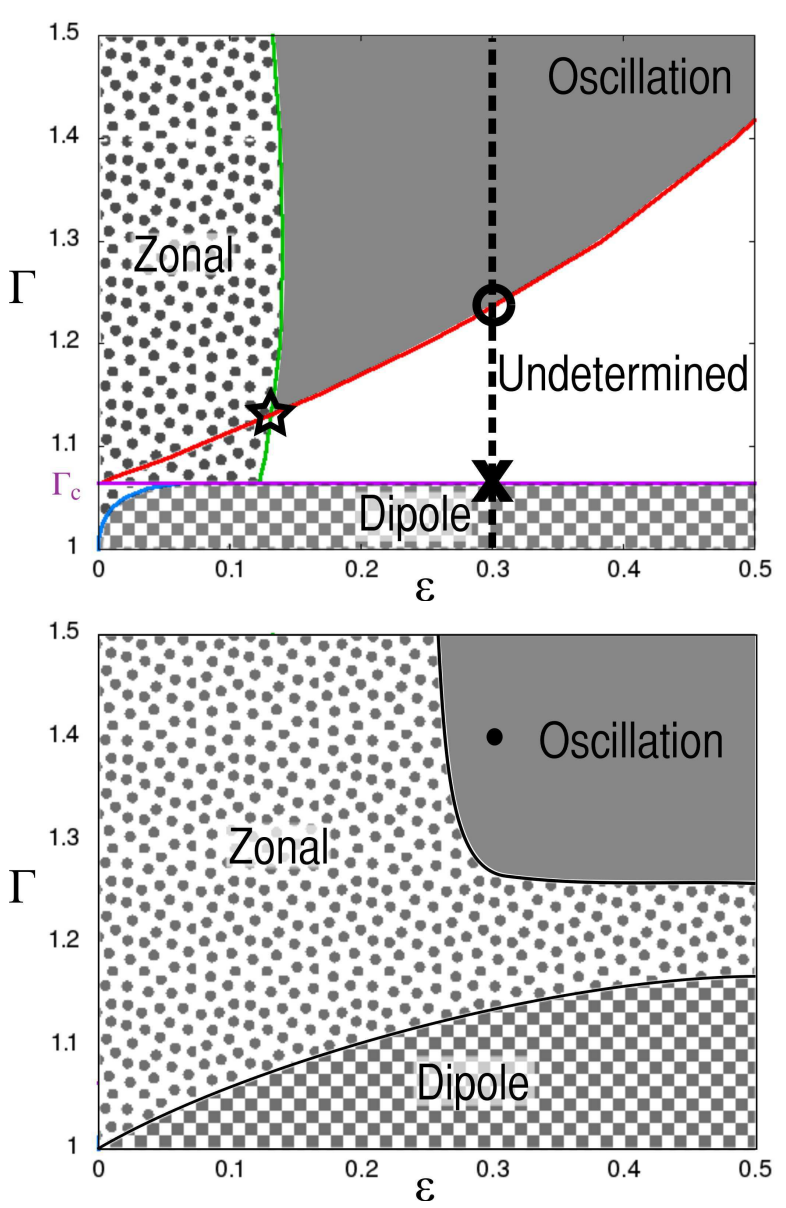

FIG. 5. (color online) Phase diagram on the $(\epsilon, \Gamma)$ plane. The gray, polka-dotted and checkered regions correspond to the oscillatory, zonal and dipole phases, respectively. The upper panel is drawn by the present theory and the lower is a schematic picture of the phase diagram obtained with the numerical simulations by Morita [17]. In the upper panel, the gray region is obtained from the inequality [52]. The left green boundary of the gray region is given by $\epsilon=\Gamma c_{\mathrm{I}}^{2} / \sqrt{1-c_{\mathrm{R}}^{2}}$ (see Eq. (18)), and the lower red by $\epsilon=\Gamma \sqrt{1-c_{\mathrm{R}}^{2}}\left(\arcsin c_{\mathrm{R}}\right)^{2}$ (see Eq. 20). The left blue boundary of the checkered region is given by $\epsilon=\Gamma c_{\mathrm{I}}^{2}$, and the upper purple by $\Gamma=\Gamma_{\mathrm{c}}$ (see Eq. [53), whose value is determined at the bifurcation point marked by the cross in Fig. 4 The star point corresponds to the star point of Fig. 4 The broken line represents $\epsilon=0.3$ on which the theoretical and numerical frequencies are compared in Fig. 7 The points marked by the cross and the circle correspond to those in Fig. 7 respectively.

the numerically computed bump positions.

Next, we obtain the frequency by computing the power spectra of the $(1,0)$ Fourier mode of $\omega(x, y, t)$ defined by

$$
Z(t)=-\operatorname{Re} \frac{1}{(2 \pi)^{2} \Gamma} \iint_{\mathbb{T}^{2}} \omega(x, y, t) e^{-i x} d x d y .
$$

As shown in Fig. 7 the dependence on $\Gamma$ of $f_{\text {num }}$ is in qualitative agreement with the prediction, in the sense that the

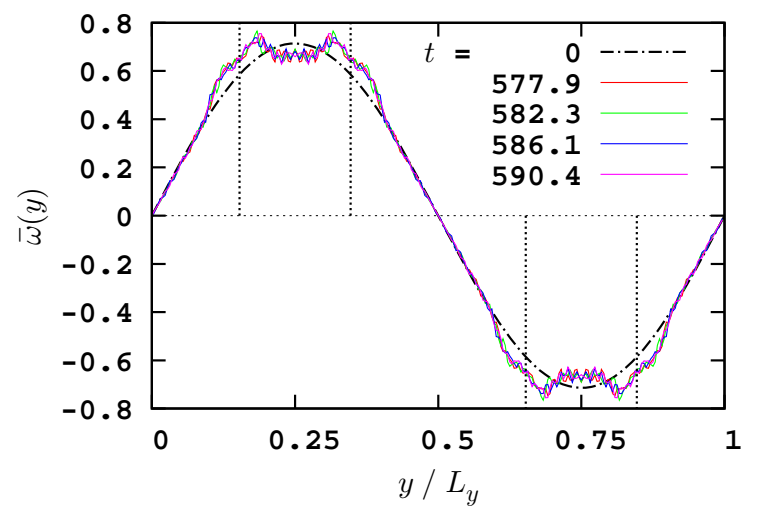

FIG. 6. (color online) The averaged vorticity field $\bar{\omega}(y, t)$. Four bumps are observed, corresponding to four vortices in the long time regime. The vertical four dotted lines indicate the theoretical predictions for the $y$-positions of the vortices: $y / L_{y}=0.25 \pm$ $0.097,0.75 \pm 0.097 . \Gamma=1.4$ and $\epsilon=0.3$.

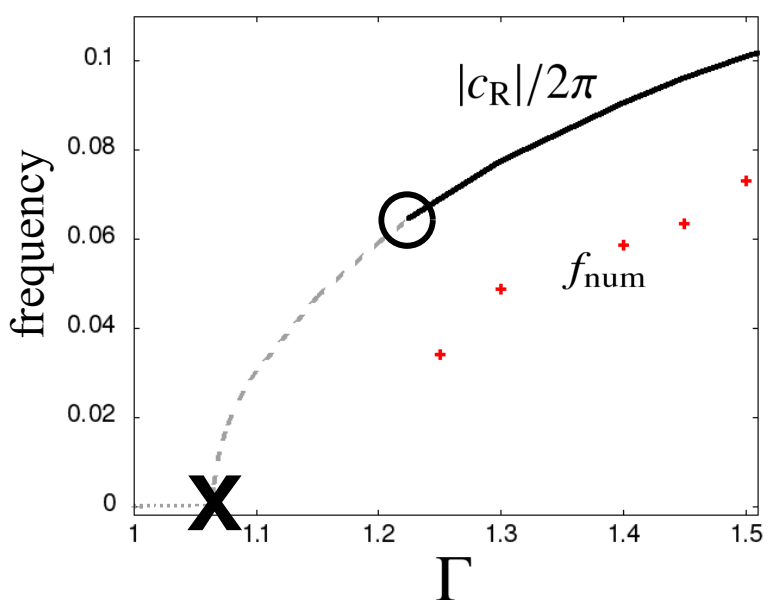

FIG. 7. (color online) $\Gamma$ dependence of the frequencies obtained from the theoretical prediction (bold curve) and the numerical simulations (red points). $\epsilon=0.3$. Clear oscillation is not found by numerical simulations in the small $\Gamma$ region, $\Gamma \lesssim 1.2$. The points marked by the cross and the circle correspond to those in Fig. 5 respectively.

frequency increases as $\Gamma$ increases. However, it is not in good agreement quantitatively. One possible explanation for the quantitative discrepancy in frequency is that $\epsilon=0.3$ is too large to be considered a small perturbation to the base flow. The present theory is based on the linear analysis of the Euler equation. It gives good predictions qualitatively, but quantitatively, nonlinear effects may kick in for rather large $\epsilon$. 


\section{SUMMARY AND DISCUSSIONS}

Inspired by previous works on the Vlasov equation 19, 22-24, 31], we have considered i) the balance between Landau damping and nonlinear effects and ii) the nonoverlapping criterion between nonlinear structures, in order to analyze the formation of a stationary dipole and traveling vortices in the 2D Euler equation starting from the perturbed stable Kolmogorov flow. The detailed linear analysis provides information on this nonlinear dynamical phenomenon. We emphasize that the bifurcation of the dominant Landau pole as the aspect ratio is varied plays a crucial role in the theory. Since this kind of bifurcation is probably not a special feature of the Kolmogorov flow, we expect that a similar analysis, predicting the appearance, or not, of a dipole or traveling vortices, would be fruitful for other base flows.

We note that our prediction is in qualitative, though not quantitative, agreement with the numerical results of Ref. [17, not only for the shape of the phase diagram, but also for the vortex frequency. The quantitative discrepancy for the phase diagram is rather natural since we have performed phenomenological order-of-magnitude estimates and not taken the prefactors into consideration. The reason for the quantitative disagreement in the frequency is probably that the size of the perturbation $\epsilon$ used in the numerical simulation is large enough to trigger nonlinear effects that we do not take into account, while such a large $\epsilon$ is necessary to realize the oscillating phase numerically.

\section{ACKNOWLEDGMENTS}

The authors are grateful to F. Bouchet for fruitful discussions and comments on this study. SO thanks for the hospitality of Université de Nice Sophia-Antipolis and acknowledges the support of the JSPS Research Fellowships for Young Scientists (Grant No. 254728). This work was supported by the ANR-09-JCJC-009401 INTERLOP project. YYY acknowledges the support of a Grant-in-Aid for Scientific Research (C) 23560069.
[1] F. Bouchet and A. Venaille, Phys. Rep. 515, 227 (2012).

[2] N. J. Balmforth, P. J. Morrison, and J. L. Thiffeault, arXiv:1303.0065

[3] P. S. Marcus, Nature 331, 693 (1988).

[4] J. B. Marston, Physics 4, 20 (2011).

[5] A. Venaille and F. Bouchet, J. Phys. Ocean. 41, 1860 (2011).

[6] W. Thomson, Nature 23, 45 (1880).

[7] H. Lamb, Hydrodynamics, 6th edition (Cambridge University Press, Cambridge, 1932).

[8] J. T. Stuart, J. Fluid Mech. 29, 417 (1967).

[9] D. Holm, J. Marsden, and T. Ratiu. in: Nonlinear systems of partial differential equations in applied mathematics, Part 2, 171-186. Series: Lectures in Appl. Math., vol. 23 (1986).

[10] Z. Lin and C. Zeng, Arch. Rational Mech. Anal. 200, 1075 (2011).

[11] L. Onsager, Nuovo Cimento. Supple. 6, 279 (1949).

[12] G. L. Eyink and K. R. Sreenivasan, Rev. Mod. Phys. 78, 87 (2006).

[13] J. Miller, Phys. Rev. Lett. 65, 2137 (1990) .

[14] J. Miller, P. B. Weichman, and M. C. Cross, Phys. Rev. A 45, 2328 (1992).

[15] R. Robert, J. Stat. Phys. 65, 531 (1991).

[16] R. Robert and J. Sommeria, J. Fluids. Mech. 229, 291 (1991).
[17] H. Morita, arXiv:1103.1140

[18] L. D. Landau, J. Phys. U.S.S.R. 10, 25 (1946); Collected papers of L. D. Landau edited by D. T. Haar (Pergamon Press, Oxford,1965).

[19] T. M. O’Neil, Phys. Fluids 8, 2255 (1965).

[20] G. Manfredi, Phys. Rev. Lett. 79, 2815 (1997).

[21] M. Brunetti, F. Califano, and F. Pegoraro, Phys. Rev. E 62, 4109 (2000).

[22] C. Lancellotti and J. J. Dorning, Phys. Rev. Lett. 81, 5137 (1998); Phys. Rev. E 68, 026406 (2003); Trans. Th. Stat. Phys. 38, 1 (2009).

[23] J. Barré and Y. Y. Yamaguchi, Phys. Rev. E 79, 036208 (2009).

[24] M. Buchanan and J. J. Dorning, Phys. Rev. Lett. 70, 3732 (1993); Phys. Rev. E 523015 (1996).

[25] R. L. Spencer and S. N. Rasband, Phys. Plasmas 4, 53 (1997).

[26] F. Bouchet and H. Morita, Physica D 239, 948 (2010).

[27] N. R. Corngold, Phys. Plasmas 2, 620 (1995).

[28] R. J. Briggs, J. D. Daugherty, and R. H. Levy, Phys. Fluids 13, 421 (1970)

[29] J. D. Crawford and P. D. Hislop, Ann. Phys. 189, 265 (1989).

[30] P. D. Hislop and J. D. Crawford, J. Math. Phys. 30, 2819 (1989).

[31] J. P. Holloway and J. J. Dorning, Phys. Rev. A 44, 3856 (1991). 